\title{
Estudio neuropsicológico del temperamento y su relación con los desórdenes de atención asociados en niños de 7 a 8 años
}

\author{
Neuropsychological study of temperament and its relationship with \\ associates attention disorders in children 7 to 8 years \\ César Sarria J1.; Victoria llaja R.; Pedro Garcia P. \\ Universidad Nacional Mayor de San Marcos, Lima, Perú \\ (RECIBIDO 10-10-2014, ACEPTADO 02-12-2014)
}

\begin{abstract}
RESUMEN
El objetivo de la presente investigación es identificar los mecanismos de deformación del sistema afectivo-emotivo y su relación con los trastornos de atención en una muestra intencional de 60 niños entre las edades de 7 a 8 años de edad, quienes cuentan con historia clínica de evaluación de control del niño sano desde los primeros meses de nacido hasta el control actual de la cohorte, en el periodo Ene-2013 a Dic-2013. Los instrumentos utilizados fueron: El SNT"(diseñado por Sarria, C. 2010) y el test de caras (Yale, 2006), a fin de medir los desórdenes atentivos de la muestra descrita, y el protocolo de evaluación de control del niño sano registrado por los especialistas pediatras del HNERM en diferentes etapas de la infancia de estos niños, de tal manera que se realizó un diseño de estudio de cohortes (Hernández, Fernández \& Baptista, 2010) en cuanto al seguimiento prospectivo de los procesos afectivoemotivos y los déficits de atención asociados. Para el análisis de los datos, se utilizó el SPSS versión 17, tablas de distribución de frecuencias y porcentajes, coeficiente de C. de Pearson y otras medidas parámetricas, que demostraron las siguientes conclusiones:
\end{abstract}

1. La interacción madre-hijo en la primera etapa de vida ejerce influencia significativa sobre la formación del sistema afectivo-emotivo y ello determinará a posteriori índices de déficit atencional.

2. Existe correlación directa entre favorable desarrollo afectivo (mostrarse contento, llorar poco en presencia de la madre) y óptimo reflejo de orientación de la atención voluntaria (vale decir, óptima recepción de los estímulos).

3. Existe correlación directa entre inadecuado desarrollo afectivo (llorar frecuentemente, incluso cuando están en brazos de su madre) y deficiente reacción de atención voluntaria hacia cambios en la estimulación (vale decir, deficiente recepción de los estímulos).

Palabras clave: Sistema, componente afectivo-emotivo, temperamento, trastorno de atención. 


\begin{abstract}
The aim of the present investigation is to identify the mechanisms of deformation of the affective - emotive system and his relation with the disorders of attention in an intentional sample of 60 children between the ages from 6 to 7 years of age, who possess clinical history of evaluation of control of the healthy child from the first months of born up to the current control of the cohort in the period in December 2011 to November 2012.The instruments will be:The SNT designed by Sarria 2010 and Faces Test : Yale, 2006 in order to measure the disorders atentivos of the described sample, and the protocol of evaluation of control of the healthy child registered by the specialists pediatricians of HNERM at different stages of the children of these children ,in such a way that will be a design of cohort study (Hernandez, Fernandez \& Baptista, 2010) with regard to the prospective follow-up of processes affective-emotional and attention deficits associated. For the analysis of the data will be used SPSS version 17, frequency distribution tables and percentages, Coefficient of Correlation Pearson and other parametric measures, showing the following conclusions:
\end{abstract}

1. The mother child interaction in the first stage of life has significant influence on the formation of emotional affective systm and this determined a posteriori indices of attention deficit.

2. There is a direct correlation between positive emotional development (load happy, mourn little in the presence of the mother and optimal orientation reflects voluntary attention (le optimal reception of stimuli).

3. There is a direct correlation between inadequate emotional development (they mourn frequently, even while in his mother arms) and poor reaction voluntary attention to changes in stimulation (le poor reception of stimuli).

Keywords: System, component affective-emotional, temperament, trastorn of attention.

\title{
INTRODUCCIÓN
}

Desde hace ya muchos años la literatura psicológica viene reportando una amplia variedad de etiologías exógenas y endógenas sobre el déficit de atención que se asocia de alguna forma a la aparición de daño estructural del cerebro; la cual sigue siendo popular y persistente. Sin embargo, la mayoría de los niños con DA no muestran historia de traumatismo en el parto y solo, en raras ocasiones, hay historias de lesiones tales como encefalitis, que podrían estar ligadas a la sintomatología clínica de los DA. Aún más, el desarrollo de cada técnica diagnóstica nueva continúa alimentando la esperanza de poder identificar alguna prueba de la hipótesis «daño cerebral». Hasta la fecha, ninguno de estos procedimientos, incluyendo la tomografía computarizada (TC), ha proporcionado datos convincentes de ninguna aberración anatómica en los niños con DA. Ortiz trata de romper el sesgo cognoscitivista y establecer una correlación con el sistema afectivo-emotivo. "El temperamento lo consideramos como el componente fundamental del sistema de la personalidad, como la forma que adopta la estructura de la personalidad desde la primeras etapas de su desarrollo formativo, es decir, en las etapas que van desde la concepción, pasando por la etapa fetal y de la infancia, hasta estruc- 
turarse socialmente como el componente afectivo del conjunto integrado de la personalidad". ( Ortiz, 1994). "Cuando decimos que un niño ha nacido inquieto, sensible, o irascible, hacemos referencia a su incipiente temperamento determinado por sensaciones afectivas que son activadas por sus necesidades más vitales. Sin duda este nivel de actividad es resultado de la primitiva codificación neural de información respecto del medio interno. No es fácil negar su importancia cuando se constata, por ejemplo, que la relativa sobreactividad del feto continúa como la hiperactividad del infante, y que su impulsividad, agresividad y emotividad puede llegar a predominar en su actividad personal" (Ortiz, 1997).

A partir de estos antecedentes, nos planteamos identificar y describir la correlación de mecanismos neurales y socioambientales que puedan relacionarse con defectos de atención y fomentar de manera precoz el desarrollo de los procedimientos de intervención adecuados.

Una amplia variedad de etiologías exógenas y endógenas se asocia a la aparición de síntomas de déficit de atención. Los trastornos por defecto de atención (TDA), que se caracterizan por impulsividad, distraimiento, periodos de atención cortos $\mathrm{y}$, algunas veces, hiperactividad, representan el trastorno de conducta más difundido en niños de edad escolar. Se ha estimado que los TDA afectan, de alguna manera, de un 3 a un $20 \%$ de la población escolar. El defecto se ha estudiado con diferentes poblaciones desde una variedad de orientaciones teóricas y metodológicas, sin llegar a resultados convincentes. La idea de que los síntomas del DA resultan de alguna forma de daño estructural del cerebro, sigue siendo popular y persistente. Sin embargo, la mayoría de los niños con DA no muestran historia de traumatismo en el parto y solo, en raras ocasiones, hay historias de lesiones tales como encefalitis, que podrían estar ligadas a la sintomatología clínica de los DA. En los últimos años, otras líneas de investigación se han abierto al debate, generando planteamientos novedosos respecto a la relación del temperamento y el apego. Dentro de este contexto, la investigación se justifica porque pretendemos conocer en mayor medida el rol de la interacción social en el periodo de máxima susceptibilidad del infante y su desarrollo afectivo como proceso subyacente en la configuración de la atención.

Asimismo, entre los objetivos específicos se hizo necesario:

1. Establecer de manera precoz indicadores pronósticos, sensibles de los defectos de atención.

2. Describir los procesos de atención subyacentes a los desórdenes afectivo-emotivos.

3. Identificar los mecanismos que puedan relacionarse en las diferencias individuales de la capacidad para atender.

Lo anteriormente señalado determinó el planteamiento del siguiente problema de investigacion: ¿Existe relación entre la formación de los procesos afectivoemotivos del temperamento y los desórdenes de atención que puedan presentarse a posteriori en niños de 7 y 8 años? 
Las hipótesis planteadas fueron:

\section{Hipótesis general}

La interacción madre-hijo y su influencia sobre la formación del sistema afectivo emotivo determinará a posteriori índices de déficit de atención.

\section{Hipótesis específicas}

1. Existe correlación directa entre favorable desarrollo afectivo (mostrarse contento, llorar poco en presencia de la madre) y óptimo reflejo de orientación de la atención voluntaria (vale decir, óptima recepción de los estímulos).

2. Existe correlación directa entre inadecuado desarrollo afectivo (llorar frecuentemente, incluso cuando están en brazos de su madre) y deficiente reacción de orientación de la atención voluntaria hacia cambios en la estimulación (vale decir, deficiente recepción de los estímulos).

\section{MÉTODO}

\section{Tipo de estudio}

La investigación fue de tipo exploratoria: estudio de cohortes. El diseño es de tipo mixto: cuanti y cualitativo, es de naturaleza descriptivo-correlacional, es decir, intenta predecir el valor aproximado de dos grupos de cohortes, 7 y 8 años, a partir del valor que tiene la variable o variables relacionadas en un momento determinado, según la clasificación de Hernández, Fernández y Baptista (2010); dirigida a una muestra de niños.

En primer lugar se tomó un breve cuestionario (SNT) sobre las condiciones del niño al nacer y sus manifestaciones clínicas. El cuestionario recoge información si "ha nacido inquieto, sensible, o irascible; haciendo referencia a su incipiente temperamento determinado por sensaciones afectivas que son activadas por sus necesidades más vitales. Sin duda este nivel de actividad es resultado de la primitiva codificación neural de información respecto del medio interno. En cuanto al seguimiento, en un segundo momento se constató, por ejemplo, que la relativa sobreactividad del feto continúa como la hiperactividad del infante, y que su impulsividad, agresividad y emotividad puede llegar a predominar en su actividad personal y que tal forma de comportamiento es punto de partida de dificultades cognitivas y de formas inadecuadas de conducta" (Ortiz, 1997).

Criterios de inclusión: niños con comprobado déficit de atención en el momento del estudio de las cohortes.

Criterios de exclusión: niños sin problemas en el temperamento desde el momento del nacimiento. 


\section{Procedimiento}

Se realizaron las coordinaciones previas con los responsables de las historias clínicas, pediatras, cuidadores, padres de los niños y el registro de control del niño sano. Se tomó en cuenta previamente los datos obtenidos en estas fichas de control(Cuestionario SNA- T) para hacer el seguimiento prospectivo de las cohortes. Los datos fueron tomados de las madres, cuando traían a sus niños a la unidad de control de niño sano los primeros años posnatales. Se hizo el seguimiento pertinente y se conformó a partir de ello 2 cohortes : de 7 y 8 años, de edad. Luego se administró el test de caras / percepción de diferencias adaptado por Yale (2006), que será correlacionado con el scanning neuropsicológico de atención/temperamento (SNA- T) (Sarria, 2010).

La evaluación se realizó en consulta externa de pediatría en sesiones de 50 a 60 minutos.

Se utilizó para el análisis de los datos el paquete estadístico SPSS versión 20.

Asimismo se desarrolló la interpretación cualitativa de resultados mediante la integración de los datos cuantitativos, a través de la propuesta de la teoría sociobiologica de la personalidad (Ortiz,1994).

\section{RESULTADOS}

Se presentan a continuación los resultados descriptivos en primer lugar y luego los datos paramétricos obtenidos en este estudio.

Tabla 1. Estadísticos del SNA-T y el test de caras.

\begin{tabular}{cccccc}
\hline \multicolumn{5}{c}{ Estadísticos descriptivos } \\
\hline Máximo & Media & Desv.típ. & $\mathrm{N}$ & Rango & Mínimo \\
\hline $\begin{array}{c}\text { SNA-T } \\
76,13\end{array}$ & 26,205 & 60 & 105 & 38 & 143 \\
$\begin{array}{c}\text { TCARAS } \\
40,27\end{array}$ & 8,342 & 60 & 30 & 22 & 52 \\
$\begin{array}{c}\text { PERCENTIL } \\
87,57\end{array}$ & 60 & 55 & 45 & 100 \\
\hline N válido (según lista) & 60 & & & \\
\hline
\end{tabular}

Correlaciones

\begin{tabular}{ll} 
& Correlaciones \\
\hline \multirow{3}{*}{ SNA-T } & SCN TCARAS \\
& Correlación de Pearson $1-, 173$ \\
& Sig. (bilateral), 186 \\
\hline TCARAS & N 6060 \\
& Correlación de Pearson -, 173 1 \\
& Sig. (bilateral) ,186 \\
& N 6060 \\
\hline
\end{tabular}


Tabla 2. Correlaciones no paramétricas.

\begin{tabular}{|c|c|c|c|c|}
\hline & & & $\mathrm{SCN}$ & PERCENTIL \\
\hline \multirow[t]{6}{*}{ Rho de Spearman } & $\mathrm{SCN}$ & $\begin{array}{l}\text { Coeficiente de } \\
\text { correlación }\end{array}$ & 1,000 &,- 176 \\
\hline & & Sig. (bilateral) & & , 180 \\
\hline & & $\mathrm{N}$ & 60 & 60 \\
\hline & PERCENTIL & $\begin{array}{l}\text { Coeficiente de } \\
\text { correlación }\end{array}$ &,- 176 & 1,000 \\
\hline & & Sig. (bilateral) &, 180 & \\
\hline & & $\mathrm{N}$ & 60 & 60 \\
\hline
\end{tabular}

Tabla 3. Correlación de Pearson.

\begin{tabular}{|c|c|c|c|c|}
\hline & & $\mathrm{SCN}$ & TCARAS & PERCENTIL \\
\hline \multirow[t]{3}{*}{$\mathrm{SCN}$} & $\begin{array}{l}\text { Correlación de } \\
\text { Pearson }\end{array}$ & 1 &, $935 * *$ &, $998 * *$ \\
\hline & Sig. (bilateral) & &, 000 &, 000 \\
\hline & $\mathrm{N}$ & 60 & 60 & 60 \\
\hline \multirow[t]{3}{*}{ T.CARAS } & $\begin{array}{l}\text { Correlación de } \\
\text { Pearson }\end{array}$ &, $935 * *$ & 1 &, $946 * *$ \\
\hline & Sig. (bilateral) &, 000 & & ,000 \\
\hline & $\mathrm{N}$ & 60 & 60 & 60 \\
\hline \multirow[t]{3}{*}{ PERCENTIL } & $\begin{array}{l}\text { Correlación de } \\
\text { Pearson }\end{array}$ &, $998 * *$ &, $946 * *$ & 1 \\
\hline & Sig. (bilateral) &, 000 &, 000 & \\
\hline & $\mathrm{N}$ & 60 & 60 & 60 \\
\hline
\end{tabular}

Tabla 4. Estadísticos descriptivos y percentiles.

\begin{tabular}{cccccccc}
\hline & N & Rango & Mínimo & Máximo & Media & Desv. típ. & Varianza \\
\hline SCN & 60 & 18 & 15 & 33 & 29,27 & 4,765 & 22,707 \\
TCARAS & 60 & 30 & 22 & 52 & 40,27 & 8,342 & 69,589 \\
PERCENTIL & 60 & 55 & 45 & 100 & 87,57 & 14,473 & 209,470 \\
N válido (según lista) & 60 & & & & & & \\
\hline
\end{tabular}


A continuación las tablas del SNA-T y el test de atención de diferencia de caras

Tabla 5. Resultados del SNA-T y test de atención.

\begin{tabular}{|c|c|c|c|c|c|c|c|}
\hline \multirow[t]{3}{*}{$N^{o}$} & \multirow[t]{3}{*}{ TEMP } & \multicolumn{2}{|c|}{$\begin{array}{l}\text { TEST DE ATENCION } \\
\text { CARITAS }\end{array}$} & \multirow[t]{3}{*}{$\mathrm{N}^{\circ}$} & \multirow[t]{3}{*}{ TEMP } & \multicolumn{2}{|c|}{$\begin{array}{c}\text { TEST DE ATENCION } \\
\text { CARITAS }\end{array}$} \\
\hline & & PTJE & PTJE & & & PTJE & PTJE \\
\hline & & DIRECTO & PERCENTIL & & & DIRECTO & PERCENTIL \\
\hline 1 & 33 & 49 & 99 & 31 & 17 & 25 & 52 \\
\hline 2 & 27 & 34 & 80 & 32 & 33 & 45 & 98 \\
\hline 3 & 33 & 49 & 99 & 33 & 33 & 46 & 98 \\
\hline 4 & 27 & 34 & 80 & 34 & 27 & 34 & 80 \\
\hline 5 & 30 & 38 & 90 & 35 & 33 & 49 & 99 \\
\hline 6 & 33 & 50 & 100 & 36 & 27 & 34 & 80 \\
\hline 7 & 17 & 24 & 50 & 37 & 30 & 38 & 90 \\
\hline 8 & 27 & 34 & 80 & 38 & 33 & 50 & 100 \\
\hline 9 & 22 & 28 & 65 & 39 & 17 & 24 & 50 \\
\hline 10 & 33 & 49 & 99 & 40 & 27 & 34 & 80 \\
\hline 11 & 32 & 44 & 97 & 41 & 22 & 28 & 65 \\
\hline 12 & 33 & 51 & 100 & 42 & 33 & 49 & X99 \\
\hline 13 & 33 & 46 & 98 & 43 & 32 & 44 & 97 \\
\hline 14 & 31 & 40 & 92 & 44 & 33 & 51 & 100 \\
\hline 15 & 33 & 49 & 99 & 45 & 33 & 46 & 98 \\
\hline 16 & 26 & 33 & 77 & 46 & 31 & 40 & 92 \\
\hline 17 & 30 & 38 & 90 & 47 & 33 & 49 & 99 \\
\hline 18 & 33 & 45 & 98 & 48 & 26 & 33 & 77 \\
\hline 19 & 33 & 45 & 98 & 49 & 30 & 38 & 90 \\
\hline 20 & 33 & 50 & 100 & 50 & 33 & 45 & 98 \\
\hline 21 & 30 & 37 & 89 & 51 & 33 & 45 & 98 \\
\hline 22 & 33 & 52 & 100 & 52 & 33 & 50 & 100 \\
\hline 23 & 25 & 32 & 75 & 53 & 30 & 37 & 89 \\
\hline 24 & 32 & 42 & 95 & 54 & 33 & 52 & 100 \\
\hline 25 & 33 & 50 & 100 & 55 & 25 & 32 & 75 \\
\hline 26 & 24 & 31 & 72 & 56 & 32 & 42 & 95 \\
\hline 27 & 25 & 32 & 75 & 57 & 33 & 50 & 100 \\
\hline 28 & 31 & 40 & 92 & 58 & 24 & 31 & 72 \\
\hline 29 & 15 & 22 & 45 & 59 & 25 & 32 & 75 \\
\hline 30 & 27 & 35 & 82 & 60 & 31 & 40 & 92 \\
\hline
\end{tabular}

La tabla $\mathrm{N}^{\circ} 5$ representa los puntajes obtenidos por los 60 sujetos de nuestra muestra, considerando los factores de temperamento y de atención (en puntajes directos y puntajes percentil). 
Tabla 6. Puntajes promedio del SNA-T y test de caras.

\begin{tabular}{ccccccc}
\hline \multicolumn{7}{c}{ Estadísticos descriptivos } \\
& $\mathrm{N}$ & Rango & Mínimo & Máximo & Media & Desv. \\
\hline & 60 & 18 & 15 & 33 & 29,27 & 4,765 \\
SCN-TEMP & 60 & 30 & 22 & 52 & 40,27 & 8,342 \\
TEST CARAS & & & & & & \\
PD & 60 & 55 & 45 & 100 & 87,57 & 14,473 \\
TEST CARAS & & & & & & \\
PPc & 60 & & & & & \\
N válido (según lista) & & & & &
\end{tabular}

En esta tabla presentamos los datos de una muestra de 60 alumnos, a quienes se les ha aplicado una escala de temperamento (SNA-T de procesos afectivo-emotivos) y un test de atención (test de caras), los siguientes datos promedio:

Respecto a su puntaje de temperamento (procesos afectivo-emotivos), este se ubica en un nivel alto (29.27); mientras que en su nivel de atención obtiene un puntaje promedio de 40.27, que para el grupo de edad lo ubica en el nivel alto. Transformando el puntaje directo a percentil, el puntaje promedio es de 87.57 para toda la muestra, lo cual representa una pertenencia al nivel de alto percentil.

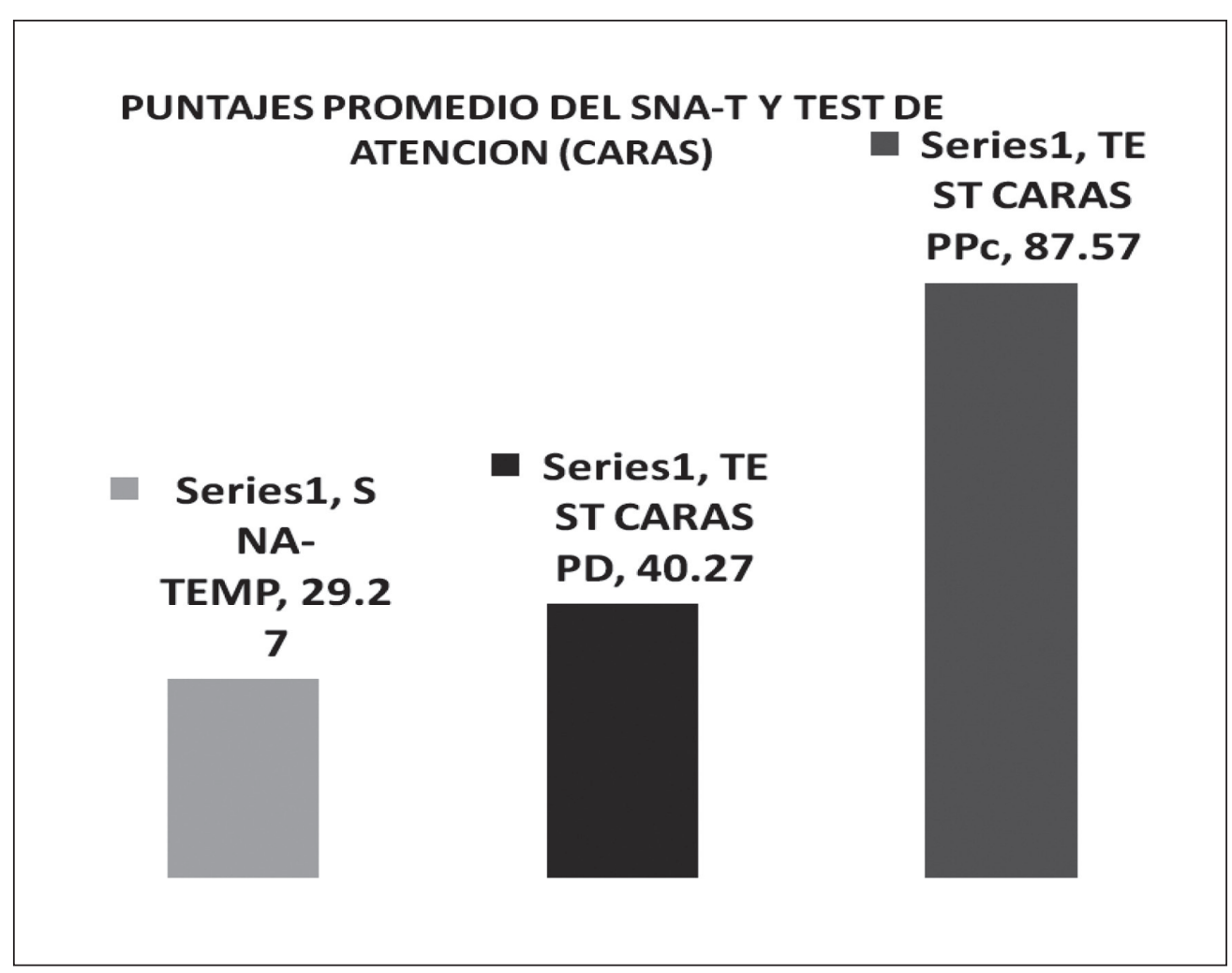


Tabla 7. Frecuencia de los niveles de atención en una muestra de alumnos del $1^{\circ}$ al $3^{\circ}$ grado de primaria

\begin{tabular}{|c|c|c|}
\hline NIVEL & Frec & $\%$ \\
\hline Promedio & 6 & \\
\hline \multicolumn{3}{|l|}{10} \\
\hline Tend. alto & 8 & \\
\hline \multicolumn{3}{|l|}{13.3} \\
\hline Alto & 46 & \\
\hline \multicolumn{3}{|l|}{76.7} \\
\hline Total & 60 & \\
\hline 100 & & \\
\hline
\end{tabular}

En esta tabla observamos que la frecuencia más alta, obtenida en el test de atención, test de caras, la frecuencia corresponde al nivel de Tendencia a alto (46.67 \%) y nivel alto $(33.3 \%)$ respectivamente. Estando ausente el nivel bajo.

Gráfica:

\section{Frecuencia de niveles de atención}

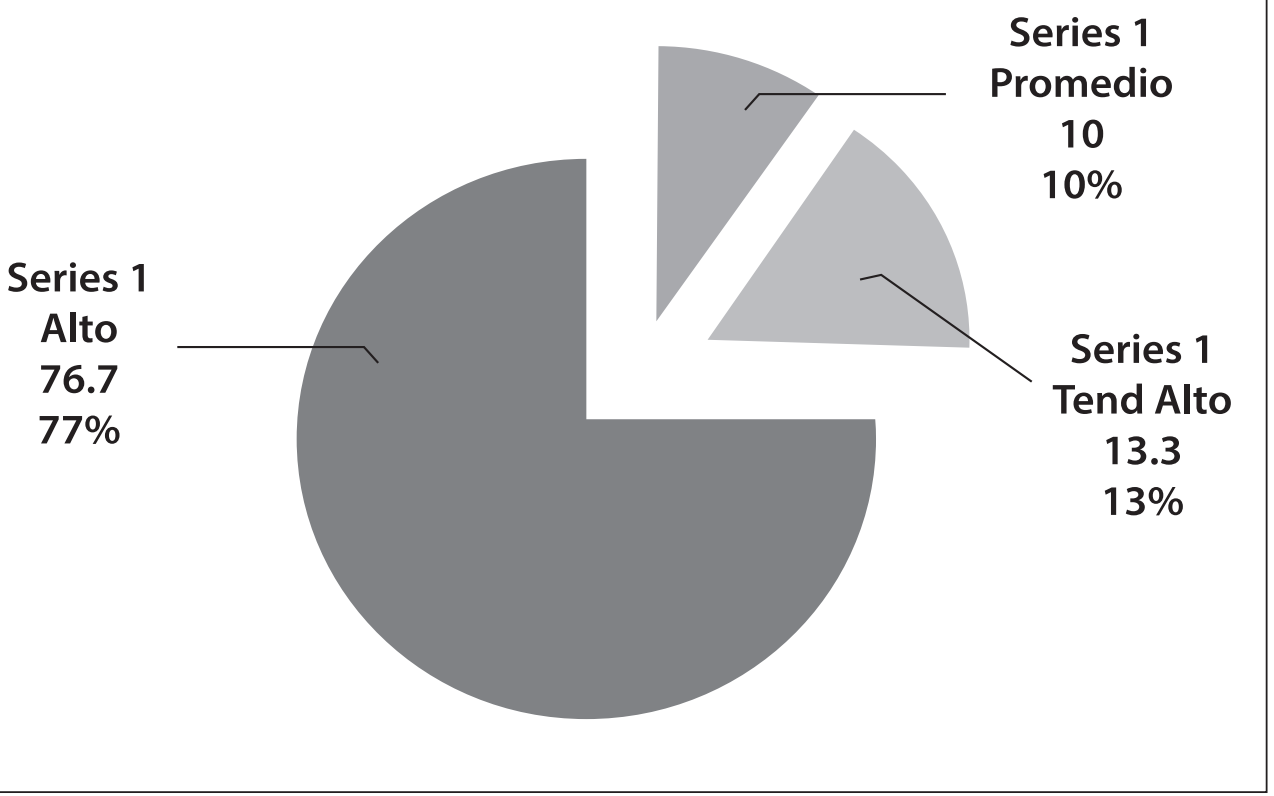

Los resultados descriptivos con su tasa de frecuencia acumulada (SNT) administrada a las madres de familia de los niños participantes en el estudio, (Tabla 8 a la Tabla 65 -ver anexos-) pueden ser vistos en el reporte ampliado de este archivo PDF. 
Tabla 8. Correlación entre SNA-T y test de caras.

\begin{tabular}{|c|c|c|c|c|}
\hline & & $\begin{array}{l}\text { SNA- } \\
\text { TEMP }\end{array}$ & $\begin{array}{c}\text { TEST } \\
\text { CARAS PD }\end{array}$ & $\begin{array}{c}\text { TEST } \\
\text { CARAS PPc }\end{array}$ \\
\hline \multirow[t]{3}{*}{ SNA-TEMP } & $\begin{array}{l}\text { Correlación de } \\
\text { Pearson }\end{array}$ & 1 &, $935 * *$ &, $998 * *$ \\
\hline & Sig. (bilateral) & & 000 & 000 \\
\hline & $\mathrm{N}$ & 60 & 60 & 60 \\
\hline \multirow[t]{3}{*}{ TEST CARAS PD } & $\begin{array}{l}\text { Correlación de } \\
\text { Pearson }\end{array}$ &, $935 * *$ & 1 &, $946 * *$ \\
\hline & Sig. (bilateral) & ,000 & & 000 \\
\hline & $\mathrm{N}$ & 60 & 60 & 60 \\
\hline \multirow[t]{3}{*}{ TEST CARAS PPc } & $\begin{array}{l}\text { Correlación de } \\
\text { Pearson }\end{array}$ & ,998** &, $946 * *$ & 1 \\
\hline & Sig. (bilateral) &, 000 &, 000 & \\
\hline & $\mathrm{N}$ & 60 & 60 & 60 \\
\hline
\end{tabular}

** La correlación es significativa al nivel 0,01 (bilateral).

En esta tabla presentamos los resultados de la correlación de 60 sujetos, considerando las variables de temperamento (procesos afectivo-emotivos) y atención. En cuanto a la correlación entre la variable temperamento y atención, el índice de correlación $\mathrm{r}$ de Pearson es de tipo positivo y muy alto (0.935); lo cual nos indica que existe un factor determinante entre ambos elementos. Este resultado nos permite aceptar las hipótesis que indican que si "existe correlación significativa entre los procesos afectivo-emotivos del temperamento y la atención”.

\section{DISCUSIÓN}

En este estudio pretendimos a través del modelo sociobiológico de la personalidad de Ortiz, P. (1997), tratar de romper el sesgo cognoscitivista y establecer la correlacion del déficit atencional con el sistema afectivo-emotivo. Dentro de este planteamiento asumimos que "el temperamento es el componente fundamental del sistema de la personalidad, como la forma que adopta la estructura de la personalidad desde la primeras etapas de su desarrollo formativo, es decir, en las etapas que van desde la concepción, pasando por la etapa fetal y de la infancia, hasta estructurarse socialmente como el componente afectivo del conjunto integrado de la personalidad". (Ortiz,1994). "Cuando postulabámos que un niño ha nacido inquieto, sensible, o irascible, hacemos referencia a su incipiente temperamento determinado por sensaciones afectivas que son activadas por sus necesidades más vitales. Sin duda, este nivel de actividad es resultado de la primitiva codificación neural de información respecto del medio interno. No es fácil negar su importancia cuando se constata, por ejemplo, que la relativa sobreactividad del feto continúa como la hiperactividad del infante, y que su impulsividad, agresividad y emotividad puede llegar a predominar en su actividad personal” (Ortiz, 1997). 
De allí que se ha podido confirmar con este protocolo de investigación que tanto el déficit de atención como la hiperactividad son solo los aspectos más objetivos del trastorno, mientras que lo primario y fundamental es un defecto en la formación del componente afectivo de la conciencia, y por lo tanto un verdadero déficit de la estructuración de la actividad anticipatoria que hemos llamado ansiedad,tal como puede comprobarse clínicamente en los pequeños desde antes de ir al jardín o al colegio Ortiz, et. al).

Los resultados encontrados son similares a los hallazgos de Jiménez (2002) que tuvo como objetivo la construcción y validación de una escala psiconeurológica en niños, aplicada a la familia, y se basó en el mismo modelo expuesto líneas arriba, en pacientes con problemas cerebrales en el servicio de Neuropsicología, del HNERM de Lima.La muestra estuvo conformada por 70 niños, que habían tenido desarrollo normal, hasta antes de la enfermedad, y la muestra no clínica fue de 120 niños con enfermedades menores. La edad promedio fue de 3 a 13 años, de ambos sexos. Las madres contaban con instrucción secundaria y superior. Se confirmó la validez de constructo y se encontraron correlaciones muy significativas en la evaluación que hicieron las madres respecto a sus hijos en las 3 áreas: comportamiento, desempeño y conducta. También existen hallazgos significativos en el estudio normativo internacional del test de caras, publicado por Ison, (2006), que estableció baremos etáreos,y que plasmaron la utilidad de este instrumento en la medición del déficit atencional, y que igualmente han vuelto a confirmar su validez y confiabilidad en nuestra muestra de estudio para la confirmación de las hipótesis planteadas en el presente reporte.

\section{CONCLUSIONES}

1. La interacción madre-hijo en la primera etapa de vida ejerce influencia significativa sobre la formación del sistema afectivo-emotivo y ello determinará a posteriori índices de déficit atencional.

2. Existe correlación directa entre favorable desarrollo afectivo (mostrarse contento, llorar poco en presencia de la madre) y óptimo reflejo de orientación de la atención voluntaria (vale decir, óptima recepción de los estímulos).

3. Existe correlación directa entre inadecuado desarrollo afectivo (llorar frecuentemente, incluso cuando están en brazos de su madre) y deficiente reacción de atención voluntaria hacia cambios en la estimulación (vale decir, deficiente recepción de los estímulos).

\section{RECOMENDACIONES}

Teniendo en cuenta la revisión de la literatura científica y los estudios previos, nos permitimos recomendar:

1. Emprender a posteriori otras investigaciones dirigidas a diversas muestras clínicas de niños que establezcan diagnósticos y pronósticos diferenciales en cuanto a los déficits de atención y su asociación con desordenes de estimulación afectiva-emotiva en el ámbito de las neurociencias y la neuropsicología aplicada. 
2. Asimismo, diseñar y administrar talleres de información dirigidos a los padres de Familia (en las escuelas de padres) con la finalidad de informar respecto a la importancia de la adecuada formación del componente del temperamento en los periodos críticos de desarrollo: los primeros 7 años de vida del niño y así adoptar medidas correctivas y preventivas de parte de ellos, a fin de estimularlos adecuadamente en esta etapa tan significativa para la formación integral de su personalidad.

\section{REFERENCIAS BIBIOGRÁFICAS}

Díaz, M. (2009). Curso Taller Avanzado. Técnicas y métodos de Investigación Científica Lima: Manual UPCH.

Hernández, Fernández \& Baptista (2010). Metodología de la Investigación. México: Mc Graw Hill.

Ison, M \& Anta, F. G. (2006). Estudio normativo del Test de Percepción de diferencias (caras) en niños Mendocinos.

Luria, A. (1980). Neuropsicología de la Atención. Madrid: Blume.

Luria, A. R. (1974). El Cerebro en Acción. Barcelona: Fontanilla.

Ortiz, C.P. (1998). El Nivel Consciente de la Memoria. Fondo Editorial de la Universidad de Lima. Lima.

Ortiz. C. P. (2004). Cuadernos de Psicobiología Social 1 Introducción a la Psicobiología del hombre. Lima: Talleres Gráficos - UNMSM.

Ortiz, C. P. (2004). Cuadernos de Psicobiología Social 6 El nivel Consciente de la Actividad Personal. Lima: talleres Gráficos - UNMSM.

Ortiz, P. (1994). El Sistema de la Personalidad. Lima: Talleres Gráficos - UNMSM.

Ortiz, P. (1996). La formación de la Personalidad. Lima: Dimaso Editores.

Ortiz. (2008). Educación y formación de la Personalidad. Lima. Fondo Editorial de la Universidad de Ciencias y Humanidades.

Reynolds, C. R. \& Hickman, J. A. (2004). Draw-A-Person Intellectual Ability Test for Children, Adolescents, and Adults (DAP:IQ). Austin: PRO-ED.

Lindgren, S. D., \&. Richman, L. D. (1984). Inmediate memory functions verbally deficient reading-disabled children. Journal of Learning Disabilities, 17, 222-225.

Richman, L. C.\& Lindgren, S.D. (1980). Patterns of intellectual ability in children withverbal deficits. Journal of Abnormal Child Psichology, 8, 65-75.

Rourke, B. P. (Ed.) (1985). Neuropsychology of learning disabilities. New York: Guilford Press.

Sarria, C, García, P., \&Llaja, V.(2008). Compendiode Neuropsicología clínica. Pesquisas Neuropsicológicas. Lima: CEPREDIM-UNMSM.

Sarria, C., García, P. \& Llaja, V. (2008). Test de Percepción Visomotor de Benton Revisado. Lima: CEPREDIM-UNMSM. 
Llaja,V. Sarria,C. García, P. (2009). Evaluación neuropsicológica del delirio y la incidencia de los factores de riesgo en pacientes de Cuidados Intensivos del HNERM. Lima: Revista de la Academia Peruana de Neurociencias del Perú. Año 1, Vol. 2, pp. 32-36.

Llaja, V, Sarria,C, García, P. (2008). Déficit cognitivos en la enfermedad de Parkinson interpretados bajo el modelo informacional de la Personalidad. Lima: Revista de la Academia Peruana de Neurociencias del Perú. Año 1, N 1, Vol. 1, pp. 25-28.

García,P. Llaja,V., Sarria,C, \& col. (julio de 2009). Aptitudes cognitivas y estrategias de memorización secuencial de los alumnos de un Centro Educativo privado de Comas. Lima: Revista de Investigación en Psicología. Instituto de Investigaciones Psicológicas de la UNMSM, Vol. 12, $\mathrm{N}^{\circ} 1$, pp. 45-59. 